\title{
THE TDHF APPROXIMATION FOR HAMILTONIANS WITH m-PARTICLE INTERACTION POTENTIALS
}

\author{
CLAUDE BARDOS*, BERNARD DUCOMET ${ }^{\dagger}$, FRANÇOIS GOLSE ${ }^{\ddagger}$, ALEX \\ D. GOTTLIEB ${ }^{\S}$, AND NORBERT J. MAUSER $₫$
}

\begin{abstract}
According to a theory of H. Spohn, the time-dependent Hartree (TDH) equation governs the 1-particle state in $N$-particle systems whose dynamics are prescribed by a non-relativistic Schrödinger equation with 2-particle interactions, in the limit $N$ tends to infinity while the strength of the 2-particle interaction potential is scaled by $1 / N$. In previous work we have considered the same mean field scaling for systems of fermions, and established that the error of the time-dependent Hartree-Fock (TDHF) approximation tends to 0 as $N$ tends to infinity. In this article we extend our results to systems of fermions with $m$-particle interactions $(m>2)$.
\end{abstract}

Key words. TDHF, BBGKY hierarchy, TDHF hierarchy, Slater closure, mean field dynamics, interacting fermions.

AMS subject classifications. 81 V70 (47N50)

\section{The TDHF equation as a mean field approximation}

The time-dependent Hartree Fock (TDHF) equation [1] is an attempt to approximate the state of a system of interacting fermions by one time-dependent Slater determinant (thus discarding any "correlation" in the many electron system, cf. [4]). In our papers $[2,3]$ we have derived the TDHF dynamics as that of a single fermion in the mean field, in the spirit of Spohn's derivation of the time-dependent Hartree equation [5] and refinements thereof $[6,7,8]$ (see [9] for a good overview). Here we show how the theorem of [2] for 2-particle interactions may be generalized to cases where the $N$-particle Hamiltonian involves $m$-particle interactions with $m>2$.

Let $\mathfrak{H}$ be a Hilbert space and let $\mathfrak{H}_{n}$ denote the $n^{\text {th }}$ tensor power of $\mathfrak{H}$, i.e.,

$$
\mathfrak{H}_{N}=\overbrace{\mathfrak{H} \otimes \mathfrak{H} \otimes \cdots \otimes \mathfrak{H}}^{n \text { times }} \text {. }
$$

For $\pi$ in the group $\mathfrak{S}_{n}$ of permutations of $\{1,2, \ldots, n\}$, define the unitary "permutation" operator $U_{\pi}$ by

$$
U_{\pi}\left(x_{1} \otimes \ldots \otimes x_{n}\right)=x_{\pi^{-1}(1)} \otimes \ldots \otimes x_{\pi^{-1}(n)}
$$

for all $x_{1}, \ldots, x_{n} \in \mathfrak{H}$. Define

$$
\mathcal{A}_{n}=\sum_{\pi \in \mathfrak{S}_{n}} \operatorname{sgn}(\pi) U_{\pi}
$$

for all $n \in \mathbb{N}$. Then $\frac{1}{n !} \mathcal{A}_{n}$ is the orthogonal projector whose range is the space of antisymmetric vectors in $\mathfrak{H}_{n}$.

\footnotetext{
${ }^{*}$ Univ Paris 7 and Laboratoire J. L. Lions (Univ. Paris 6), France (bardos@math.jussieu.fr).

${ }^{\dagger}$ CEA/DAM Ile de France, DPTA/Service de Physique Nucléaire, BP 12, F-91680 Bruyères-leChâtel, France (bernard.ducomet@cea.fr).

${ }^{\ddagger}$ Laboratoire J. L. Lions (Univ. Paris 6), France (golse@math.jussieu.fr).

$\S$ Wolfgang Pauli Inst., Nordbergstr. 15, A-1090 Wien, Austria (alex@alexgottlieb.com).

๑WPI c/o Fak. f. Math., Univ. Wien, Nordbergstr. 15, A-1090 Wien, Austria (mauser@courant. nyu.edu).
} 
Consider $N$ identical fermions whose 1-particle Hilbert space is $\mathfrak{H}$. The appropriate $N$-fermion Hilbert space is the space of antisymmetric wavefunctions in $\mathfrak{H}_{N}$, i.e., the range of the orthogonal projector $\frac{1}{N !} \mathcal{A}_{N}$. If $\left\{e_{j}\right\}_{j \in J}$ is an orthonormal basis of $\mathfrak{H}$ then the set

$$
\left\{\frac{1}{\sqrt{N !}} \mathcal{A}_{N}\left(e_{j_{1}} \otimes e_{j_{2}} \otimes \cdots \otimes e_{j_{N}}\right):\left\{j_{1}, j_{2}, \ldots, j_{N}\right\} \subset J\right\}
$$

is an orthonormal basis of the antisymmetric subspace of $\mathfrak{H}_{N}$. Antisymmetric vectors built in this way are called Slater determinants.

The statistical states of the $N$-fermion system are density operators, i.e., nonnegative operators of trace 1 , that are supported on the antisymmetric subspace of $\mathfrak{H}_{N}$. These "fermionic" densities are those density operators $D$ on $\mathfrak{H}_{N}$ that satisfy

$$
D U_{\pi}=U_{\pi} D=\operatorname{sgn}(\pi) D \quad \forall \pi \in \mathfrak{S}_{N} .
$$

A density operator $D$ on $\mathfrak{H}_{N}$ that commutes with every permutation operator $U_{\pi}$ is symmetric; fermionic density operators are a special sort of symmetric density operators.

The evolution of the state of the system, as given by the $N$-particle density operator $D_{N}(t)$, is governed by a von Neumann equation

$$
i \frac{d}{d t} D_{N}(t)=\left[H_{N}, D_{N}(t)\right]
$$

where $H_{N}$ denotes the Hamiltonian operator on $\mathfrak{H}_{N}$ for the $N$-particle system. If $H_{N}$ commutes with the permutation operators $U_{\pi}$ for all $\pi \in \mathfrak{S}_{N}$, then $D_{N}(t)$ remains fermionic (resp., symmetric) at all $t>0$ if $D_{N}(0)$ was fermionic (resp., symmetric). We consider $H_{N}$ that are sums of symmetric $k$-particle interaction potentials for $k \leq m$, where $m>2$ is arbitrary but finite, and fixed in the limit $N \longrightarrow \infty$. To be specific, using the notation

$$
X^{[k]}=X(X-1) \cdots(X-k+1)
$$

for the " $k^{\text {th }}$ factorial power" of a number $X$, we consider

$$
H_{N}=\sum_{1 \leq j \leq N} L_{j}+\frac{1}{N-1} \sum_{1 \leq i<j \leq N} V_{i j}^{(2)}+\cdots+\frac{1}{(N-1)^{[m-1]}} \sum_{1 \leq i_{1}<\cdots<i_{m} \leq N} V_{i_{1} i_{2} \ldots i_{m}}^{(m)},
$$

where the 1-particle energy operator $L$ and the $k$-particle interaction potentials $V^{(k)}$ are defined as follows. $L$ denotes a self-adjoint operator on the 1 -particle space $\mathfrak{H}$, e.g., the kinetic energy operator plus an external potential, and

$$
L_{j}=\overbrace{I \otimes \cdots \otimes I}^{j-1 \text { times }} \otimes L \otimes \overbrace{I \otimes \cdots \otimes I}^{N-j \text { times }}
$$

for $j=1, \ldots, N$. For $2 \leq k \leq m$, the $k$-particle interaction potential $V^{(k)}$ is assumed to be a bounded operator on $\mathfrak{H}_{k}$ for $k=1, \cdots, m$ that commutes with all $U_{\pi}$ for $\pi \in \mathfrak{S}_{k}$; and the operator $V_{i_{1} i_{2} \ldots i_{k}}^{(k)}$ on $\mathfrak{H}_{N}$ is defined to equal $U_{\pi}^{*} V_{1,2, \ldots, k}^{(m)} U_{\pi}$, where $\pi$ is any permutation in $\mathfrak{S}_{N}$ with $j=\pi\left(i_{j}\right)$ for $j=1, \ldots, m$, and

$$
V_{1,2, \ldots, k}^{(k)}\left(x_{1} \otimes \cdots \otimes x_{N}\right)=V^{(k)}\left(x_{1} \otimes \cdots \otimes x_{k}\right) \otimes x_{k+1} \otimes \cdots \otimes x_{N} .
$$


In (1.4), the $k$-particle potentials are scaled by 1 over the $(k-1)^{t h}$ factorial power of $(N-1)$. This coefficient is asymptotically the same as $N^{-k}$ in the limit $N \longrightarrow \infty$ that concerns us, but the precise choice of $1 /(N-1)^{[k-1]}$ for the coefficients is made so that $H_{N}$ may be written as

$$
H_{N}=\sum_{1 \leq j \leq N} L_{j}+\frac{1}{(N-1)^{[m-1]}} \sum_{1 \leq i_{1}<\cdots<i_{m} \leq N} W_{i_{1} i_{2} \ldots i_{m}},
$$

with

$$
W_{1,2, \ldots, m}=\sum_{k=2}^{m}(m-k) ! \sum_{1 \leq j_{1}<\cdots<j_{k} \leq m} V_{j_{1} j_{2} \ldots j_{k}}^{(k)}
$$

Naturally, the sum of single-particle potentials $L_{j}$ could be included in the definition (1.6) of the effective $m$-particle potential $W$, but we must treat the single-particle operators $L_{j}$ separately because we do not assume that they are bounded, while we do assume that all the higher interaction potentials $V^{(k)}$ are bounded (and therefore $H_{N}$ is a bounded perturbation of the free Hamiltonian).

The TDHF approximation is in fact an approximation of the 1-particle density operator $D_{N: 1}(t)$ obtained from $D_{N}(t)$ by taking a partial trace. Partial traces may be defined as follows. Let $\mathcal{O}$ be any orthonormal basis of $\mathfrak{H}$. When $T$ is any trace class operator on $\mathfrak{H}_{N}$ and $n<N$, the partial trace $T_{: n}$ of $T$ is the operator on $\mathfrak{H}_{n}$ such that

$$
\left\langle y, T_{: n} x\right\rangle=\sum_{j=1}^{N-n} \sum_{z_{j} \in \mathcal{O}}\left\langle y \otimes z_{1} \otimes \cdots \otimes z_{N-n}, T\left(x \otimes z_{1} \otimes \cdots \otimes z_{N-n}\right)\right\rangle
$$

for any $x, y \in \mathfrak{H}^{\otimes n}$ (this quantity is independent of the choice of $\mathcal{O}$ ). The partial trace takes fermionic densities to fermionic densities: if a trace class operator $T$ satisfies (1.2) then so does $T_{: n}$.

The time-dependent Hartree-Fock (TDHF) equation corresponding to (1.5) is

$$
i \frac{d}{d t} F(t)=[L, F(t)]+\frac{1}{(m-1) !}\left[W, F(t)^{\otimes m} \mathcal{A}_{m}\right]_{: 1}
$$

(here $[X, Y]_{: 1}$ denotes the partial trace of the commutator of $X$ and $Y$ ). Following [10], we define a strong solution of equation of (1.8) to be a continuously differentiable function $F(t)$ from $[0, \infty)$ to the real Banach space of Hermitian trace class operators such that the domain of $L$ is invariant under $F(t)$ for all $t \geq 0$ and

$$
i \frac{d F(t)}{d t} x=[L, F(t)] x+\frac{1}{(m-1) !}\left[W, F(t)^{\otimes m} \mathcal{A}_{m}\right]_{: 1} x
$$

for all $x$ in the domain of $L$. A straightforward extension of the results proved in [10] shows that (1.8) has a strong solution if the domain of $L$ contains the range of the initial condition $F(0)$. Furthermore, $F(t)=U^{*} F(0) U$ for some unitary operator depending on $t$ and $F(0)$. In particular, the operator norm and the trace norm of $F(t)$ are constant.

Theorem 1.2 below states that the error of the TDHF approximation of $D_{N: 1}(t)$ tends to 0 as $N \longrightarrow \infty$. Theorem 1.2 contains further information about the $n$ particle density operators $D_{N: n}(t)$, expressed in terms of the concept of "Slater closure" defined in [2]. Slater closure is analogous to the condition of asymptotic chaos as 
conceived by Mark Kac [11], i.e., the attainment in the limit $N \longrightarrow \infty$ of Boltzmann's "molecular chaos" (stochastic independence of particles). Theorem 1.2 confirms that something analogous to the "propagation of chaos" is valid for mean field fermion systems. If $P_{\Psi_{N}}$ denotes the orthogonal projector onto the span of a Slater determinant $\Psi_{N}$, then

$$
\left(P_{\Psi_{N}}\right)_{: n}=\frac{N^{n}}{N^{[n]}}\left(P_{\Psi_{N}}\right)_{: 1}^{\otimes n} \mathcal{A}_{N} ;
$$

this motivates the following definition of Slater closure:

Definition 1.1. For each $N$, let $D_{N}$ be a symmetric density operator on $\mathfrak{H}_{N}$ and let $\mathcal{A}_{n}$ be the projector defined in (1.1). The sequence $\left\{D_{N}\right\}$ has Slater closure if, for each fixed $n$,

$$
\lim _{N \rightarrow \infty}\left\|D_{N: n}-D_{N: 1}{ }^{\otimes n} \mathcal{A}_{n}\right\|_{1}=0 .
$$

TheOREM 1.2. For each $N$, let $D_{N}(t)$ be a solution of (1.3) whose initial value $D_{N}(0)$ is a symmetric density operator, and let $F_{N}(t)$ be the solution of the TDHF equation (1.8) whose initial value is $F_{N}(0)=D_{N: 1}(0)$.

If $\left\{D_{N}(0)\right\}$ has Slater closure then $\left\{D_{N}(t)\right\}$ has Slater closure and

$$
\lim _{N \rightarrow \infty}\left\|D_{N: 1}(t)-F_{N}(t)\right\|_{1}=0
$$

for all $t>0$.

\section{Sketch of proof of theorem $\mathbf{1 . 2}$}

The proof of Theorem 1.2 closely follows the proof in [2].

The idea is to compare the BBGKY hierarchy to the so-called TDHF hierarchy. The BBGKY hierarchy is the system of equations satisfied by $D_{N: 1}, D_{N: 2}, \ldots$ when $D_{N}$ satisfies the Schrödinger equation (1.3) and all $D_{N: n}$ for $n>N$ are defined to be identically 0 . The TDHF hierarchy is the system of equations satisfied by $F, F^{\otimes 2} \mathcal{A}_{2}, F^{\otimes 3} \mathcal{A}_{3}$, etc. when $F$ satisfies the TDHF equation (1.8). The partial trace $D_{N: n}(t)$ satisfies

$$
\begin{aligned}
i \frac{d}{d t} D_{N: n}(t)= & \sum_{j=1}^{n}\left[L_{j}, D_{N: n}(t)\right]+\frac{1}{(m-1) !} \sum_{j=1}^{n}\left[W_{j, n+1, \ldots, n+m-1}, D_{N: n+m-1}(t)\right]_{: n} \\
& +\mathcal{E}_{n}\left(D_{N}(t)\right),
\end{aligned}
$$

where the error term $\mathcal{E}_{n}\left(D_{N}(t)\right)$ is small in trace norm when $N$ is large relative to $n$. If $F(t)$ is a strong solution of the TDHF equation (1.8) then

$$
\begin{aligned}
i \frac{d}{d t} F(t)^{\otimes n} \mathcal{A}_{n}= & \sum_{j=1}^{n}\left[L_{j}, F(t)^{\otimes n} \mathcal{A}_{n}\right] \\
& +\frac{1}{(m-1) !} \sum_{j=1}^{n}\left[W_{j, n+1, \ldots, n+m-1}, F(t)^{\otimes n+m-1} \mathcal{A}_{n+m-1}\right]_{: n} \\
& +\mathcal{R}_{n}(F(t)),
\end{aligned}
$$

where $\mathcal{R}_{n}$ is likewise small in trace norm when $N>>n$. One sees that the sequences $\left\{D_{N: n}(t)\right\}_{n}$ and $\left\{F(t)^{\otimes n} \mathcal{A}_{n}\right\}$ each satisfy a hierarchy of equations, which are the same 
up to terms that are small in trace norm. From equations (2.1) and (2.2) it follows that $E_{N, n}(t) \equiv D_{N: n}(t)-F_{N}(t)^{\otimes n} \mathcal{A}_{n}$ satisfies

$$
\begin{aligned}
i \frac{d}{d t} E_{N, n}(t)= & \sum_{j=1}^{n}\left[L_{j}, E_{N, n}(t)\right]_{: n}+\frac{1}{(m-1) !} \sum_{j=1}^{n}\left[W_{j, n+1, \ldots, n+m-1}, E_{N, n+m-1}\right]_{: n} \\
& +\mathcal{E}_{n}\left(D_{N}(t)\right)-\mathcal{R}_{n}\left(F_{N}(t)\right)
\end{aligned}
$$

for $n=1,2, \ldots, N-1$.

Now we claim that, for each fixed $n \in \mathbb{N}$, the trace norms of the extra terms $\mathcal{E}_{n}\left(D_{N}(t)\right)$ and $\mathcal{R}_{n}\left(F_{N}(t)\right)$ in $(2.1)$ and $(2.2)$, and hence the difference $\mathcal{E}_{n}\left(D_{N}(t)\right)$ $\mathcal{R}_{n}\left(F_{N}(t)\right)$ of these errors in (2.3), are $O(1 / N)$ as $N \longrightarrow \infty$ uniformly in $t$. To see that $\left\|\mathcal{E}_{n}\left(D_{N}(t)\right)\right\|_{1}=O(1 / N)$, let us enumerate the terms of the form $\left[W_{i_{1} i_{2} \ldots i_{m}}, D_{N}(t)\right]_{: n}$ that arise when one takes the $n^{\text {th }}$ partial trace of both sides of the von Neumann equation (1.3) with $H_{N}$ given by (1.5), counting by the number of indices $i_{1}, \ldots, i_{m}$ that are larger than $n$. When all of these indices are larger than $n$, the $n^{\text {th }}$ partial trace of the commutator equals 0 . When all indices except $i_{1}$ are in $\{1, \ldots, n\}$, the terms $\left[W_{i_{1} i_{2} \ldots i_{m}}, D_{N}(t)\right]_{: n}$ all equal $\left[W_{i_{1}, n+1, \ldots, n+m-1}, D_{N}(t)\right]_{: n}$ by the symmetry of $D_{N}$. The sum of the partial traces of these terms appears on the right-hand side of (2.1), if

$$
\left(\frac{\left(\begin{array}{c}
N-n \\
m-1
\end{array}\right)}{(N-1)^{[m-1]}}-\frac{1}{(m-1) !}\right) \sum_{j=1}^{n}\left[W_{j, n+1, \ldots, n+m-1}, D_{N: n+m-1}(t)\right]_{: n},
$$

is counted separately as part of the error $\mathcal{E}_{n}\left(D_{N}(t)\right)$. This contribution to $\mathcal{E}_{n}$ is bounded by

$$
\frac{2\|W\|}{(m-1) !}\left(\frac{(N-n)^{[m-1]}}{(N-1)^{[m-1]}}-1\right)
$$

in trace norm, and this is $O(1 / N)$. The rest of the terms $\left[W_{i_{1} i_{2} \ldots i_{m}}, D_{N}(t)\right]_{: n}$, those for which $i_{2} \in\{1, \ldots, n\}$, also belong to the error $\mathcal{E}_{n}$. Each of these terms is bounded in trace norm by $2\|W\|$, and the number of them is $O\left(N^{m-2}\right)$, so their contribution to $\mathcal{E}_{n}$ is $O(1 / N)$. The proof that $\left\|\mathcal{R}_{n}\left(F_{N}(t)\right)\right\|_{1}=O(1 / N)$ is deferred for the moment; for now we complete the proof, supposing that there do exist bounds $f_{n}(N)$ such that

$$
\left\|\mathcal{E}_{n}\left(D_{N}(t)\right)-\mathcal{R}_{n}\left(F_{N}(t)\right)\right\|_{1} \leq f_{n}(N)
$$

uniformly in $t$, and such that $f_{n}(N)=O(1 / N)$ as $N \longrightarrow \infty$ for each fixed $n$.

It is convenient to rewrite (2.3) in the "interaction picture," whereby the generator $-i \sum\left[L_{j}, \cdot\right]$ of the unperturbed (free) dynamics is eliminated from the right-hand side of the equation, while all operators on the right-hand side assume the dependence on time used in the Heisenberg picture for the free dynamics. Changing to the interaction picture does not change the trace norm of the error term in $(2.3)$, for $-i \sum\left[L_{j}, \cdot\right]$ generates a unitary group of isometries of the space of trace class operators. The details of this transformation are discussed in Section 4 of [2]. Here we get the estimate

$$
\left\|E_{N, n}(t)\right\|_{1} \leq\left\|E_{N, n}(0)\right\|_{1}+f_{n}(N) t+b n \int_{0}^{t}\left\|E_{N, n+m^{\prime}}(s)\right\|_{1} d s,
$$


where $b=2\|W\| /(m-1) !, m^{\prime} \equiv m-1$, and $f_{n}(N)$ is as in (2.4). Iterate (2.5) $k$ times to obtain the bound

$$
\begin{aligned}
\left\|E_{N, n}(t)\right\|_{1} \leq & \sum_{j=0}^{k}(b t)^{j} \frac{n\left(n+m^{\prime}\right) \cdots\left(n+(j-1) m^{\prime}\right)}{j !}\left\|E_{N, n+j m^{\prime}}(0)\right\|_{1} \\
& +\sum_{j=0}^{k} b^{j} t^{j+1} \frac{n\left(n+m^{\prime}\right) \cdots\left(n+(j-1) m^{\prime}\right)}{(j+1) !} f_{n+j m^{\prime}}(N) \\
& +(b t)^{k+1} n\left(n+m^{\prime}\right) \cdots\left(n+k m^{\prime}\right) \\
& \times \int_{0}^{t} \int_{0}^{s_{1}} \cdots \int_{0}^{s_{k}}\left\|E_{N, n+(k+1) m^{\prime}}(s)\right\|_{1} d s_{k} \cdots d s_{1} d t .
\end{aligned}
$$

The last term on the right-hand side of the preceding equation is bounded by

$$
2 \frac{n\left(n+m^{\prime}\right) \cdots\left(n+k m^{\prime}\right)}{(k+1) !}(b t)^{k+1}
$$

since the trace norm of $E_{N, n+(k+1) m^{\prime}}(s)$ can never be larger than 2 (see Lemma 5.2 of [2]). As long as $t<1 / b,(2.8)$ may be made arbitrarily small by choosing $k$ large enough. On the other hand, for fixed $k$, the sums (2.6) and (2.7) tend to 0 as $N \longrightarrow \infty$. The terms of (2.7) tend to 0 because of our claim that $f_{n}(N)=O(1 / N)$, and the terms of (2.6) tend to 0 because

$$
\begin{aligned}
\lim _{N \rightarrow \infty}\left\|E_{N, p}(0)\right\|_{1} & \equiv \lim _{N \rightarrow \infty}\left\|D_{N: p}(0)-F_{N}(0)^{\otimes p} \mathcal{A}_{p}\right\|_{1} \\
& =\lim _{N \rightarrow \infty}\left\|D_{N: p}(0)-D_{N: 1}(0)^{\otimes p} \mathcal{A}_{p}\right\|_{1}=0
\end{aligned}
$$

for all $p \in \mathbb{N}$ by the hypotheses of Theorem 1.2. By choosing $k$ arbitrarily large and letting $N \longrightarrow \infty$, it can be shown that

$$
\lim _{N \rightarrow \infty}\left\|E_{N, n}(t)\right\|_{1} \equiv \lim _{N \rightarrow \infty}\left\|D_{N: p}(t)-F_{N}(t)^{\otimes p} \mathcal{A}_{p}\right\|_{1}=0
$$

as long as $t<1 / b$. This proves the theorem for $t<1 / b$. The argument can be iterated to establish the theorem for all $t$, as described in Section 6 of [2].

Finally, we return to the proof that $\left\|\mathcal{R}_{n}\left(F_{N}(t)\right)\right\|_{1}=O(1 / N)$. In fact, strong solutions of the TDHF equation (1.8) satisfy

$$
\begin{aligned}
i \frac{d}{d t} F_{N}(t)^{\otimes n} \mathcal{A}_{n}= & \sum_{j=1}^{n}\left[L_{j}, F_{N}(t)^{\otimes n} \mathcal{A}_{n}\right] \\
& +\frac{1}{(m-1) !} \sum_{j=1}^{n}\left[W_{j, n+1, \ldots, p}, F_{N}(t)^{\otimes p} \mathcal{A}_{\{j, n+1, \ldots, p\}} \mathcal{A}_{n}\right]_{: n}
\end{aligned}
$$

exactly, where

$$
p=n+m-1
$$

and $\mathcal{A}_{\{j, n+1, \ldots, p\}}$ equals the sum of $\operatorname{sgn}(\pi) U_{\pi}$ as in (1.1), but the sum is only over permutations $\pi \in \mathfrak{S}_{p}$ such that $\pi(x)=x$ for all $x \notin\{j, n+1, \ldots, p\}$. Thus $\mathcal{R}_{n}\left(F_{N}(t)\right)$ in $(2.2)$ is

$$
\frac{1}{(m-1) !} \sum_{j=1}^{n}\left[W_{j, n+1, \ldots, n+m-1}, F_{N}(t)^{\otimes p}\left(\mathcal{A}_{\{j, n+1, \ldots, n+m-1\}} \mathcal{A}_{n}-\mathcal{A}_{n+m-1}\right)\right]_{: n},
$$


and it follows that

$$
\begin{aligned}
\left\|\mathcal{R}_{n}\left(F_{N}(t)\right)\right\|_{1} & \leq \frac{n}{(m-1) !}\left\|\left[W_{n, n+1, \ldots, p}, F_{N}(t)^{\otimes p}\left(\mathcal{A}_{\{n, n+1, \ldots, p\}} \mathcal{A}_{n}-\mathcal{A}_{n+m-1}\right)\right]_{: n}\right\|_{1} \\
& \leq \frac{2 n}{(m-1) !}\left\|\left\{W_{n, n+1, \ldots, p} F_{N}(t)^{\otimes p}\left(\mathcal{A}_{\{n, n+1, \ldots, p\}} \mathcal{A}_{n}-\mathcal{A}_{p}\right)\right\}_{: n}\right\|_{1} .
\end{aligned}
$$

When both $J \subset\{1, \ldots, n\}$ and $K \subset\{n+1, \ldots, p\}$ have the same number $\ell$ of elements, let $U_{(J K)}$ denote the permutation operator

$$
U_{(J K)} \equiv U_{\left(j_{1} k_{1}\right)\left(j_{2} k_{2}\right) \cdots\left(j_{\ell} k_{\ell}\right)},
$$

where $j_{1}<j_{2}<\cdots<j_{\ell}$ are the elements of $J$ and $k_{1}<\cdots<k_{\ell}$ are the elements of $K$. With this notation we can write the identity

$$
\mathcal{A}_{p}=\mathcal{A}_{\{n+1, \ldots, p\}}\left(I+\sum_{\ell=1}^{\min \{n, m-1\}}(-1)^{\ell} \sum_{\substack{J \subset\{1, \ldots, n\} \\ \# J=\ell}} \sum_{\substack{K \subset\{n+1, \ldots, p\} \\ \# K=\ell}} U_{(J K)}\right) \mathcal{A}_{n} .
$$

Subtracting this identity from the identity

$$
\mathcal{A}_{\{n, n+1, \ldots, p\}} \mathcal{A}_{n}=\mathcal{A}_{\{n+1, \ldots, p\}}\left(I-\sum_{k=n+1}^{p} U_{(n k)}\right) \mathcal{A}_{n}
$$

we find that

$$
\mathcal{A}_{\{n, n+1, \ldots, p\}} \mathcal{A}_{n}-\mathcal{A}_{p}=\mathcal{A}_{\{n+1, \ldots, p\}} \mathcal{B A}_{n}
$$

where

$$
\mathcal{B}=-\sum_{k=n+1}^{p} U_{(n k)}-\sum_{\ell=1}^{\min \{n, m-1\}}(-1)^{\ell} \sum_{\substack{J \subset\{1, \ldots, n\} \\ \# J=\ell}} \sum_{\substack{K \subset\{n+1, \ldots, p\} \\ \# K=\ell}} U_{(J K)}
$$

Substituting (2.12) in (2.11), we find that

$$
\begin{aligned}
\left\|\mathcal{R}_{n}\left(F_{N}(t)\right)\right\|_{1} & \leq \frac{2 n}{(m-1) !}\left\|\left\{W_{n, n+1, \ldots, p} F_{N}(t)^{\otimes p} \mathcal{A}_{\{n+1, \ldots, p\}} \mathcal{B} \mathcal{A}_{n}\right\}_{: n}\right\|_{1} \\
& =\frac{2 n}{(m-1) !}\left\|\left\{W_{n, n+1, \ldots, p} \mathcal{A}_{\{n+1, \ldots, p\}} F_{N}(t)^{\otimes p} \mathcal{B}\right\}_{: n} \mathcal{A}_{n}\right\|_{1} \\
& \leq \frac{2 n ! n}{(m-1) !}\left\|\left\{W_{n, n+1, \ldots, p} \mathcal{A}_{\{n+1, \ldots, p\}} F_{N}(t)^{\otimes p} \mathcal{B}\right\}_{: n}\right\|_{1} .
\end{aligned}
$$

The last inequality in (2.14) holds by the triangle inequality and the fact that $\|T U\|_{1}=$ $\|T\|_{1}$ for all unitary $U$ and trace class $T$, and the equality preceding it holds because $F_{N}(t)^{\otimes p}$ commutes with $\mathcal{A}_{\{n+1, \ldots, p\}}$. Substitute (2.13) into (2.14) and apply the triangle inequality; it results that $\left\|\mathcal{R}_{n}\left(F_{N}(t)\right)\right\|_{1}$ is bounded by a sum of terms of the form

$$
\frac{2 n ! n}{(m-1) !}\left\|\left\{W_{n, n+1, \ldots, p} \mathcal{A}_{\{n+1, \ldots, p\}} U_{\left(j_{2} k_{2}\right) \cdots\left(j_{\ell} k_{\ell}\right)} F_{N}(t)^{\otimes p} U_{\left(j_{1} k_{1}\right)}\right\}_{: n}\right\|_{1},
$$


where $j_{1}<n$ and $U_{\left(j_{2} k_{2}\right) \cdots\left(j_{\ell} k_{\ell}\right)}$ denotes $I$ in case $\ell=1$ (note that $F_{N}(t)^{\otimes p}$ commutes with any permutation operator $\left.U_{(j k)}\right)$. We will show that each of these terms is $O(1 / N)$. Let $M_{n, \ldots, p}$ denote $W_{n, n+1, \ldots, p} \mathcal{A}_{\{n+1, \ldots, p\}}$. Then

$$
\begin{aligned}
(2.15) & =\frac{2 n ! n}{(m-1) !}\left\|\left\{M_{n, \ldots, p} U_{\left(j_{2} k_{2}\right) \cdots\left(j_{\ell} k_{\ell}\right)} F_{N}(t)^{\otimes p} U_{\left(j_{1} k_{1}\right)}\right\}_{: n}\right\|_{1} \\
& =\frac{2 n ! n}{(m-1) !}\left\|\left\{M_{n, \ldots, p} U_{(2, p-1) \cdots(\ell, p-\ell+1)} F_{N}(t)^{\otimes p} U_{(1, p)}\right\}_{: n}\right\|_{1} \\
& \leq \frac{2 n ! n}{(m-1) !}\left\|\left\{M_{n, \ldots, p} U_{(2, p-1) \cdots(\ell, p-\ell+1)} F_{N}(t)^{\otimes p} U_{(1, p)}\right\}_{: p-1}\right\|_{1} \\
& =\frac{2 n ! n}{(m-1) !} \|\left(F_{N}(t) \otimes I^{\otimes p-2}\right) U_{(1,2, \cdots, p-1)} M_{n-1, \ldots, p-1} \\
& \leq \frac{2 n ! n}{(m-1) !}\left\|F_{N}(t)\right\|\|M\|\left\|F_{N}(t)^{\otimes p-1}\right\|_{1} \\
& \leq 2 n ! n\|W\|\left\|F_{N}(t)\right\| \\
& \leq 2 n ! n\|W\| \frac{1}{N} .
\end{aligned}
$$

Inequality holds in $(2.16)$ holds because $X_{: n}=\left(X_{: p-1}\right)_{: n}$, and the trace norm of a partial trace is less than or equal to the trace norm of the original operator. In equation (2.17) — which is verified using the definition (1.7) of the partial trace $(1,2, \cdots, p-1)$ denotes the permutation

$$
\left(\begin{array}{ccccc}
1 & 2 & \cdots & p-2 & p-1 \\
2 & 3 & \cdots & p-1 & 1
\end{array}\right)
$$

and $(p-1, p-2, \ldots, 1)$ denotes its inverse. Inequality (2.18) comes from applying the general bound $\|B T\|_{1} \leq\|B\|\|T\|_{1}$, valid for all bounded operators $B$ and trace class operators $T$, and inequality (2.19) holds because $\left\|F_{N}(t)\right\|_{1}=\left\|F_{N}(0)\right\|_{1}=$ $\left\|D_{N: 1}(0)\right\|_{1}=1$. Finally, inequality $(2.20)$ holds because $\left\|F_{N}(t)\right\|=\left\|F_{N}(0)\right\|=$ $\left\|D_{N: 1}(0)\right\|$, and $\left\|D_{N: 1}\right\| \leq 1 / N$ if $D_{N}$ is an $N$-particle fermionic density operator.

Acknowledgement. This work was supported by the Austrian Ministry of Science (BM:BWK) via its grant for the Wolfgang Pauli Institute and by the Austrian Science Foundation (FWF) via the START Project (Y-137-TEC) of N. Mauser, and also by the European network HYKE funded by the EC as contract HPRN-CT-200200282.

Also, F. G. was supported by the Institut Universitaire de France. The first four authors thank the WPI and the ESI in Vienna for its hospitality.

\section{REFERENCES}

[1] P. A. M. Dirac, Note on exchange phenomena in the Thomas atom, Proc. Cambridge Phil. Soc., 26, 376-385, 1930.

[2] C. Bardos, F. Golse, A. D. Gottlieb and N. J. Mauser, Mean field dynamics of fermions and the time-dependent Hartree-Fock equation, J. de Math. Pures et Appl., 82, 665-683, 2003.

[3] C. Bardos, F. Golse, A. D. Gottlieb and N. J. Mauser, Accuracy of the time-dependent HartreeFock approximation for uncorrelated initial states, J. Stat. Phys., 115, 1037-1055, 2004. 
[4] A. D. Gottlieb and N. J. Mauser, New measure of electron correlation, Phys. Rev. Lett., 95(12), 213-217, 2005

[5] H. Spohn, Kinetic equations from Hamiltonian dynamics, Rev. Mod. Phys., 53, 600-640, 1980

[6] C. Bardos, F. Golse and N. J. Mauser, Weak coupling limit of the N-particle Schrödinger equation, Math. Anal. Appl., 7(2), 275-293, 2000.

[7] C. Bardos, L. Erdös, F. Golse, N. J. Mauser and H.-T. Yau, Derivation of the SchrödingerPoisson equation from the quantum N-particle Coulomb problem, C. R. Acad. Sci., t 334(6), Série I Math., 515-520, 2002.

[8] L. Erdös and H.-T. Yau, Derivation of the nonlinear Schrödinger equation from a many body Coulomb system, Adv. Theor. Math. Phys., 5, 1169-1205, 2001.

[9] P. Gérard, Equations de champ moyen pour la dynamique quantique d'un grand nombre de particules, [d'après Bardos, Erdös, Golse, Gottlieb, Mauser, Yau], Séminaire Bourbaki 56ème année, 2003-2004, n ${ }^{0} 930$.

[10] A. Bove, G. Da Prato and G. Fano, On the Hartree-Fock time-dependent problem, Comm. Math. Phys., 49, 25-33, 1976.

[11] M. Kac, Foundations of kinetic theory, Proceedings of the Third Berkeley Symposium on Mathematical Statistics and Probability, Vol III. University of California Press, Berkeley, California, 1956 